4. R. V. Kadison, Isomorphisms of factors of infinite type, Canad. J. Math. 7 (1955), 322-327. MR 17, 178.

5. R. I. Loebl and P. S. Muhly, Analyticity and flows on von Neumann algebras (in preparation).

6. M. Takesaki, Duality for crossed products and the structure of von Neumann algebras of type III, Acta Math. 131 (1973), 249-310.

DEPARTMENT OF MATHEMATICS, WAYNE STATE UNIVERSITY, DETROIT, MICHIGAN 48202

DIVISION OF MATHEMATICAL SCIENCES, UNIVERSITY OF IOWA, IOWA CITY, IOWA 52242

BULLETIN OF THE

AMERICAN MATHEMATICAL SOCIETY

Volume 81 , Number 4 , July 1975

\title{
INTEGRAL TRANSFORMS OF WEAK TYPE BETWEEN REARRANGEMENT INVARIANT SPACES
}

BY MARIO MILMAN

Communicated March 20, 1975

1. Introduction. Let $X(\Omega), Y(\Omega)$ and $Z(\Omega \times \Omega)$ be rearrangement invariant Banach function spaces, where $\Omega=(0, \infty)$ with the Lebesgue measure. Let $M(\Omega)$ be the set of measurable functions on $\Omega$ and for every $k \in Z(\Omega \times \Omega)$, denote by $z_{k}$ the integral operator given by $z_{k}(f)(x)=\int_{\Omega} k(x, y) f(y) d y$ for $f \in M(\Omega), x \in \Omega$.

In this paper we shall give necessary and sufficient conditions, in terms of the fundamental functions of the spaces (see [2] and [4]) for $z_{k}$ to be of weak type $\{X, Y\}$ for every $k \in Z$. The methods are similar to those employed by $O$ 'Neil in his fundamental paper [3].

2. The Lorentz $\Lambda(Z)$ and $M(Z)$ spaces. It is well known how to define the Lorentz $\Lambda$ and $M$ spaces associated with $X(\Omega)$. To extend these definitions to $Z(\Omega \times \Omega)$, we "smash" $Z$ into $\hat{Z}(\Omega)$, say, via Luxemburg's representation theorem [1]. The relationship between the fundamental functions of these 
spaces is $\phi_{Z}(t, s)=\phi_{\hat{Z}}(t . s)$. We define $\Lambda(Z)(\Omega \times \Omega)$ to be $\Lambda_{\phi \hat{Z}}(\Omega \times \Omega)$ and $M(Z)(\Omega \times \Omega)=M_{\phi \hat{Z}}(\Omega \times \Omega)$.

3. Results. Our first theorem is a generalization of a result obtained by O'Neil [3, p. 217], where only Orlicz spaces were considered.

THEOREM 1. Suppose there exists a constant $c>0$ such that

$$
s \phi_{Y}(t) \leqslant c \phi_{Z^{\prime}}(t . s) \phi_{X}(s) \quad \forall t, s>0 .
$$

Then we have:

(i) $\left\|z_{k}(f)\right\|_{M(Y)} \leqslant$ const $\|k\|_{M(Z)}\|f\|_{\Lambda(X)}$,

(ii) $\left\|z_{k}(f)\right\|_{M(Y)} \leqslant$ const $\|k\|_{\Lambda(Z)}\|f\|_{M(X)}$,

(iii) $\left\|z_{k}(f)\right\|_{\Lambda(Y)} \leqslant$ const $\|k\|_{\Lambda(Z)}\|f\|_{\Lambda(X)}$.

TheOREM 2. Suppose that $Y \in U$. (See [2] and [4].) Then $z_{k}$ is of weak type $\{X, Y\}$ for every $k \in M(Z)$ if and only if condition (1) is verified.

Finally, using interpolation, we have

THEOREM 3. If condition (1) is verified, then $\forall k \in \Lambda(Z)_{b} z_{k}$ is a bounded operator from $\Lambda_{\alpha}(X)$ to $\Lambda_{\beta}(Y)$ where $\beta<\alpha$.

(For the definition and properties of the $\Lambda_{\alpha}(X)$ spaces we refer the reader to [2] and [4] .)

Detailed proofs will appear elsewhere.

\section{REFERENCES}

1. W. A. J. Luxemburg, Rearrangement-invariant Banach function spaces, Queen's Papers in Pure and Appl. Math., no. 10, Queen's University, Kingston, Ont., 1967, pp. 83-144.

2. M. Milman, Convolution operators and $\Lambda_{\alpha}\left(L_{\rho}\right)$ spaces (preprint).

3. R. O'Neil, Integral transforms and tensor products on Orlicz spaces and $L(p, q)$ spaces, J. Analyse Math. 21 (1968), 1-276.

4. R. Sharpley, Spaces $\Lambda_{\alpha}(X)$ and interpolation, J. Functional Analysis 11 (1972), 479-513.

DEPARTMENT OF PURE MATHEMATICS, SCHOOL OF GENERAL STUDIES, AUSTRALIAN NATIONAL UNIVERSITY, CANBERRA, AUSTRALIA 